\title{
Contactless power transfer system for low power medical devices
}

\author{
Leong Kah Meng ${ }^{a}$, Tan Tian Swee a, ${ }^{,}$, Sameen Ahmed Malik ${ }^{a}$, Tan Jia Hou ${ }^{a}$, Tengku Ahmad \\ Iskandar Tengku Alang ${ }^{\mathrm{a}}$, Lum Kin Yun ${ }^{\mathrm{b}}$
}

Medical Device and Technology Group, IJN-UTM Cardiovascular Engineering Centre, Faculty of Bioscience and Medical Engineering, Universiti
Teknologi Malaysia, 81310 Skudai, Malaysia
Department of Electrical and Electronic Engineering Faculty of Engineering and Built Environment Tunku Abdul Rahman University College Jalan
Genting Kelang, Setapak, 53300, Kuala Lumpur.

* Corresponding author: tantswee@biomedical.utm.my

\section{Article history}

Received 29 September 2017

Accepted 7 December 2017

\begin{abstract}
Currently, contactless power transfer system is being implemented to power up the medical devices. Those medical devices can either power by transcutaneous cable or batteries. However, the power driven for medical devices via transcutaneous cable might lead to infection due to breach on the skin. The battery replacement surgery for cardiovascular patients also can lead to infection. Therefore, a contactless power transfer system is effectively solving the problem by transmitting power in a safe and non-invasive manner and has mitigate effect to patient health, yet efficient in the power transmission process. Magnetic coupled resonant is being designed since magnetic field cannot be shielded by biological tissues. Resonant power transfer technique can minimize the power scattering on the non-resonant objects such as human body. Impedance matching technique is utilized to improve the overall power transmission efficiency. The system consists a pair of transmitter and receiver coil, transmitter and receiver circuit, and a pair of transformer as impedance matching purpose. The system is capable to achieved a transfer efficiency of over $60 \%$. Contactless power transfer technology does offer the advantages of safety, non-invasive and no significant effect on patient health. Eventually, bacterial infection on the skin breach is prevented.
\end{abstract}

Keywords: Wireless power transfer, contactless energy transfer, impedance matching, transformer, transfer efficiency

(c) 2017 Penerbit UTM Press. All rights reserved

\section{INTRODUCTION}

The energy supply is crucial in the functionality of medical devices. Hence, an efficient, continuous and stable supply of energy is required. These devices must receive energy supply externally or harvest the ambient energy. However, the batteries in implantable medical devices can only be replaced or recharged by surgery. An alternative method to use is transcutaneous power cable. Generally, the percutaneous link across the body skin is used to recharge the internal battery (RamRakhyani et al., 2011). This may pose infection risks to the patients (Samad et al., 2006 \& Si, P., et al., 2007). The power cord also restricts the patient to peform activities related to water. Therefore, an alternative solution such as wireless power charging may be a viable solution (Chandrakasan et al., 2008).

This system is expected to contribute to the advancement of biomedical technology for more patients to benefit from it especially for those who need the biomedical devices to sustain their life. The increasing usability of cables for charging purposes towards the biomedical devices will limit the mobility of patients during the charging process. In addition, when replacing the batteries of some implantable biomedical devices in human body, it will increase risk of several infection during the operation surgery. Hence, the wireless power charging system is a better solution for those problems.

Magnetic resonant coupling is being proposed to apply into midrange wireless power transfer application. Magnetic resonant coupling is the near field wireless transmission of electrical energy between two magnetically coupled coils of wire are resonant at the same frequency. This phenomenon occurs when a resonant transformer, and electrical component which consist of two high quality factor coils act as transmitter and receiver materials and connected across the winding to make two coupled inductance and capacitance (LC) circuits. This type of resonant technique are widely used as band pass filter, switching power supplies and wireless power systems. There are two separate LC circuit with several devices. First part consist of transmitter coil which transmits electric power across an intervening space to a resonant receiver coil. The receiver coil connected to the load or other devices. The technology was researched for powering and charging the electronic devices such as mobile phones, laptop, tablet computer and so on at a distance without wire. The advantage of using this magnetic resonant coupling transfer technique is to improve the transfer efficiency. When this technology is used, a pair of primary and secondary coils which act as transmitter and receiver is capacitively loaded to form tuned LC circuit. If the transmitter and receiver coils are resonant at the same frequency, the power can be transmitted between the coils over a range of the transmitter coils diameter with reasonable efficiency. In addition, if any obstables (non-magnetic materials) is placed between the transmitter and receiver, the transmitted power will still can pass through the obstable without and power lose.

Wireless power transfer can be achieved using magnetic field couplings that are non-radiative (Hui et al., 2014). On the other way, the radiation emmited can be negligible. These mutual coupling 
between transmitter and receiver circuit has been research over decade (Mur-Miranda et al., 2010; Imura et al., 2011; Liu et al., 2017). Transcutaneous energy transmission (TET) system has been introduce by a group of researchers to power LVAD (Slaughter et al., 2010; Wang et al., 2013). The technique used during for the TET system are inductive coupling within transmitter and receiver coils. The size of the transmitter and receiver are $5 \mathrm{~cm}$. The TET system was able to power 10 watt across a $1 \mathrm{~cm}$ distance with a efficiency of 65 percent. During the experiment, the temperature of the transmitter and receiver coils increase. Therefore, it is not suitable to use for implantable device.

Four coils wireless power transfer system refer to the wireless power system consist of transmitter and receiver coils pair (two coils wireless power system) and added two coil resonators into the system (Hui et al., 2014). This two coil resonator aid as the input and output impedance matching function for the wireless transfer syste in order to achieve the maximum transfer efficiency. Free-Range Resonant Electrical Energy Delivery (FREE-D) system is introduced by a group of researchers in year 2012 (Waters et al., 2012 \& 2014). The FREED system adopted the four coils wireless power transfer system to power LVAD. The frequency used during the experiment was $13.56 \mathrm{MHz}$. It can transfer $70 \mathrm{~cm}$ with a 75 percent of transfer efficiency. The transmitter and receiver coils size are $9.5 \mathrm{~cm}$ and the resonator coils used are $59 \mathrm{~cm}$.

Two coils wireless power transfer system consist of transmitter and receiver coils. Four coils wireless power transfer system is modify from two coils wireless power transfer system, and sending resonator and receiving resonator were added in between the transmitter and receiver coils. However four coils wireless power transfer system is more complex than two coils wireless power transfer system due to the mutual coupling coefficient within the pairs of coils. Four coils wireless power transfer system consist more coils than two coils wireless power transfer system. The distance of resonators put between the transmitter and receiver coils had to be fixed due to the frequency tunning technique used achieved maximum power transfer. But two coils wireless power transfer system are flexible within the transmission range. Other than that, more coils on the system result the frequency splitting phenomenon occur often. It will affect the transfer efficiency of the system.

In order to solve the safety problem regarding the wireless power transfer, a group of researchers face the problem regarding the electromagnetic field issues in the four coil wireless tranfer system. The electric field and magnetic field were measured when the power of 60 watt had been transfer from transmitter coil to reveiver coil with frequency of $10 \mathrm{MHz}$ at the distance of 2 meter (Bawa et al, 2008 \& Badr et al, 2017). The electric field measurement was recorded at the middle of the two resonators which electric field $=210 \mathrm{~V} / \mathrm{m}$ and magnetic field $=1 \mathrm{~A} / \mathrm{m}$. The researcher repearted the measurement at the point of $20 \mathrm{~cm}$ near the resonator coil surface and recorded the reading. It shows that the value for electric field and magnetic field gradually increased. Now the new electric field $=1400 \mathrm{~V} / \mathrm{m}$ and magnetic field $=8 \mathrm{~A} / \mathrm{m}$. The new reading has higher than expected value as the regulation stated. In order to fed the IEEE regulations, the researchers modify the coil and reduce the operating frequency from $10 \mathrm{MHz}$ to $1 \mathrm{MHz}$. This caused the transmission range for the system decreases. Therefore, the low resonance frequency which lower than $1 \mathrm{MkHz}$ was chosen for this contactless power transfer system for safety reason.

Wireless power transfer system with relay resonators transfer power wirelessly with aid of relay resonators. These relay resonators were place between the transmitter coil and receiver coil. Without any impedance matching method, this system is workeble. It can transfer power wirelessly at a frequency of $15 \mathrm{kHz}$ with the helps of relay resonators. Other than that, this wireless power transfer with relay resonators is more suitable to use for transmitting signal and low power devices with high frequency which exceeding $10 \mathrm{MHz}$. It is transmited through a series of planar printed resonators and magnetoinductive waveguide (Syms, et al., 2010). The impedance matching method used in the magneto-inductive waveguide is follow the maximum power transfer theorem. So,the energy transfer efficiency transfer by the system of the magneto-inductive waveguide usually lower 50 percent. This relay resonator system between transmitter coil and receiver coil was then tested and improved with energy transfer sfficiency and increased transmission distance (Zhang, F. et al. 2010 $\&$ 2011). These projects high depend on high frequency operation which using frequencies up to megahertz and it is also adopt the maximum power theorem. During a high frequency operation, it required an RF amplifier as the power source. For the low power application, this system with relay resonators had been proposed to open up a body sensor network technology for health care and medical propose research.

Wireless power domino resonator systems were modified from the magneto-inductive waveguide concept and it was being research recently. This wireless power domino resonator systems was a very flexible systems. It combine with the various number resonators to be place in several domino forms (Lee, C. K. et al., 2012; Zhong, W. X. et al., 2012). It is different with the mageto-inductive waveguide which required to be operate at high frequency. This wireless power domino resonator systems had been sucessfully tested at frequency of $500 \mathrm{kHz}$, under a near-filed magnetic coupling and maximum energy efficiency priciples was applied to the system. During year 2013, some research regarding the general analysis on the wireless power domino resonator system had been reported. The analyes of space optimizing of resonators, operating frequencies and load to achieve maximum energy transfer efficiency have been researched (Zhong, W. et al., 2013). During the experiment, the researchers found out that for the short distance between the resonators can achieved stronger mutual coupling with high energy efficiency during the wireless power domino resonator systems. They also tested the wireless power domino resonator systems with different patterns of arrangement for examples, straight-line, curved, circular and Y-shape. The advantage of this wirelss power domino resonator systems is that the power flow will be follow by the arrangement of the domino patterns of shapes. Therefore we can said this system was very flexible of controling the direction of the power flow. In addition, the power flow can be either split or combined, it is highlgy depend on the resonator arrangement. Although it could provide a flexible power path for wireless transfer system, but the research for this wirelss power domino resonators system is still in the initial stage. Due to the mulitple coil-resonators used in the system, the wrong coupling with the non-adjacent resonators become an issue during the power transfered. This wrong cross coupling of the resonators results the frequency switching away from the original resonance frequency of the coil resonators. This domino system can be applied to high power application because it operate or adopte the maximum energy transfer efficiency principle.

The research for these four types of wireless transfer technologies had become a trend in the field of wireless power transfer. The common on these four type of wireless power transfer technique are the mutual coupling resonance between the coil in order to transfer the power wirelessly. Table 1 shows the comparison between the four types of magnetic resonant coupling techniques used for wireless power transfer.

Table 1 Comparison for four types of magnetic resonant coupling techniques.

\begin{tabular}{|c|c|c|c|c|}
\hline & $\begin{array}{l}\text { Two Coils } \\
\text { System }\end{array}$ & $\begin{array}{l}\text { Four } \\
\text { Coils } \\
\text { System }\end{array}$ & $\begin{array}{c}\text { Relay } \\
\text { Resonators } \\
\text { System }\end{array}$ & $\begin{array}{l}\text { Dominor } \\
\text { Resonator } \\
\text { s System }\end{array}$ \\
\hline Resonators & No & Two & Multiples & Multiples \\
\hline Technique & $\begin{array}{l}\text { Impedance } \\
\text { matching }\end{array}$ & $\begin{array}{l}\text { Frequenc } \\
\text { y Tunning }\end{array}$ & No & No \\
\hline Efficiency & $\geq 65 \%$ & $\geq 65 \%$ & $50 \% \leq$ & $50 \% \leq$ \\
\hline
\end{tabular}

Two coils wireless power transfer system consist of transmitter and receiver coils. Four coils wireless power transfer system is modify from two coils wireless power transfer system, and sending resonator 
and receiving resonator were added in between the transmitter and receiver coils. However four coils wireless power transfer system is more complex than two coils wireless power transfer system due to the mutual coupling coefficient within the pairs of coils. Four coils wireless power transfer system consist more coils than two coils wireless power transfer system. The distance of resonators put between the transmitter and receiver coils had to be fixed due to the frequency tunning technique used achieved maximum power transfer. But two coils wireless power transfer system are flexible within the transmission range. Other than that, more coils on the system result the frequency splitting phenomenon occur often. It will affect the transfer efficiency of the system.

Wireless power transfer system with relay resonators can transfer power wirelessly from transmitter to receiver coil with the aid of coil resonators. Wireless power domino resonator systems were modified from the wireless power transfer system with relay resonators. It also consist of resonators in between the transmitter and receiver coils. However, the wireless power domino resonator systems is more flexible than wireless power transfer system with relay resonators. It can control the power flow path with the arrangement of the resonators at certain domino patterns. These two wireless power transfer system had a common disadvantages. When more resonators used in the wireless power transfer system, the power will be great reduce through the resonators. It reuslt a low transfer effeciency during the wireless power transfer system.

\section{MATERIALS AND METHOD}

\section{Hardware Prototype}

This system will be divided into two main parts. First is the transmitter circuit and second is the receiver circuit. In order to increase the efficiency the system, a impedance matching method by adding two transformers into the system was implemented. A step-up transformer is added into the transmitter circuit and a step-down transformer is added into the receiver circuit. This impedance matching method ensures that system always operate in its best performance. In designing this receiving circuit, space constraint is another issue besides the power limitation. The size of the receiving module is reducing, leading to the decreased space available for the receiving coils. As a result, the frequency of the power transmission has to be increased proportionally.

\section{Impedance Matching}

A impedance matching method is designed by adding the transformer into the two coil wireless power transfer system. As mention in the introduction, the most suitable way to implement this project is the two coil system. Therefore, an analysis of two coil system with and without transformer was carry out using Matlab. The analysis aims to maximize the transfer efficiency of the contactless power transfer system. Several conditions were considering into the analysis which is the coupling coefficients between the coils, critical coupling point between two resonant coils and purely resistive impedance for transmistter and reveiver circuit. At the end of the analysis results into two simplify equations which represent the stepup and step-down transformer gain in below (Leong K. M. et al., 2016):

$$
\begin{gathered}
n_{2}=\sqrt{\frac{L_{1} R_{1} R_{2}}{R_{L}\left(L_{1} R_{2}+L_{2} R_{1}\right)}} \\
n_{1}=n_{2} \sqrt{\frac{1}{2}\left(\frac{L_{1}}{L_{2}}\right)}\left(\frac{R_{L}}{R_{S}}\right)
\end{gathered}
$$

Rs is the source impedance and $\mathrm{RL}_{\mathrm{L}}$ is the load. $\mathrm{R}_{1}$ and $\mathrm{R}_{2}$ are the parallel resistance of the resonant coils on the transmitting side and receiving side respectively. L1 and L2 are the inductance value for the transmitting side and receiving side. $\mathrm{n} 1$ represent the step-up transformer gain and $n_{2}$ represent the step-down transformer gain in the system. Equation (1) and (2) were then apply into the transformer impedance modeling to optimize efficiency of the system.

Fig. 1 shows the steps to implement the transformer base impedance modeling into the two coil wireless power transfer system.

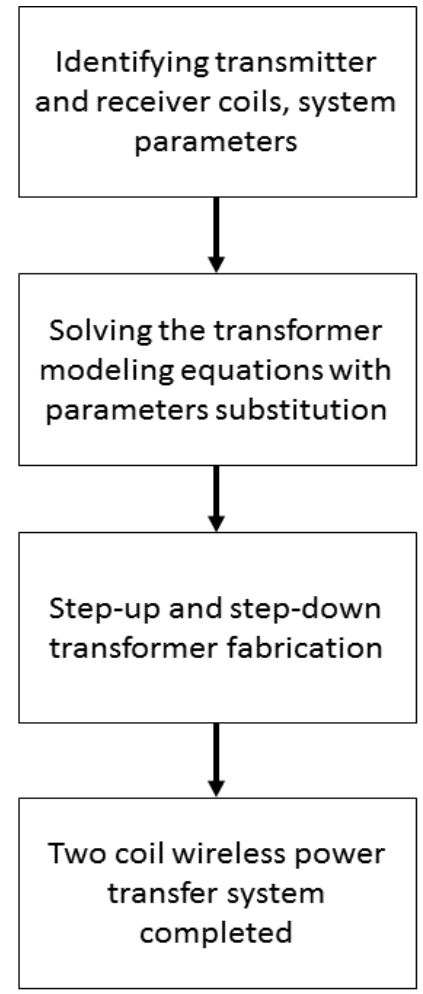

Fig. 1 Flows for transformer impedance modeling.

Firstly, the quality factor, resistance, inductance of the transmitter and receiver coils were measured using LCR meter. The resistance and the inductance of the transmiter and receiver circuit will also be measured. After identified the parameters, the parameters are then substituted into the equations (1) and (2). n1 represent the step-up transformer and $n_{2}$ represent the step-down transformer in the system. Once the values of $n_{1}$ and $n_{2}$ is obtained, the transformer can be fabricated. When the step-up transformer and step-down tranformer are adding to the transmitter and receiver cricuit, the two coil wireless power transfer system is completed.

\section{Software Simulation}

A simulation was carry out to verify the concept which the system with impedance matching technique will provide a better transfer efficiency and incresed transfer distance. The simulation was simulated using Agilent Advance Design System (ADS). Both schematic diagram with impedance matching and without impedance matching were draw and the simulated results were compared. Fig. 2 illustrates the schematic for simulation in Agilent Advance Design System for both unmatch two coil and matched two coil WPT system. This simulation intend to verify the system with impedance matching will increased the transfer distance of the system. The results for both schematic diagram were simulate and further elaborated in section results and discussion. 


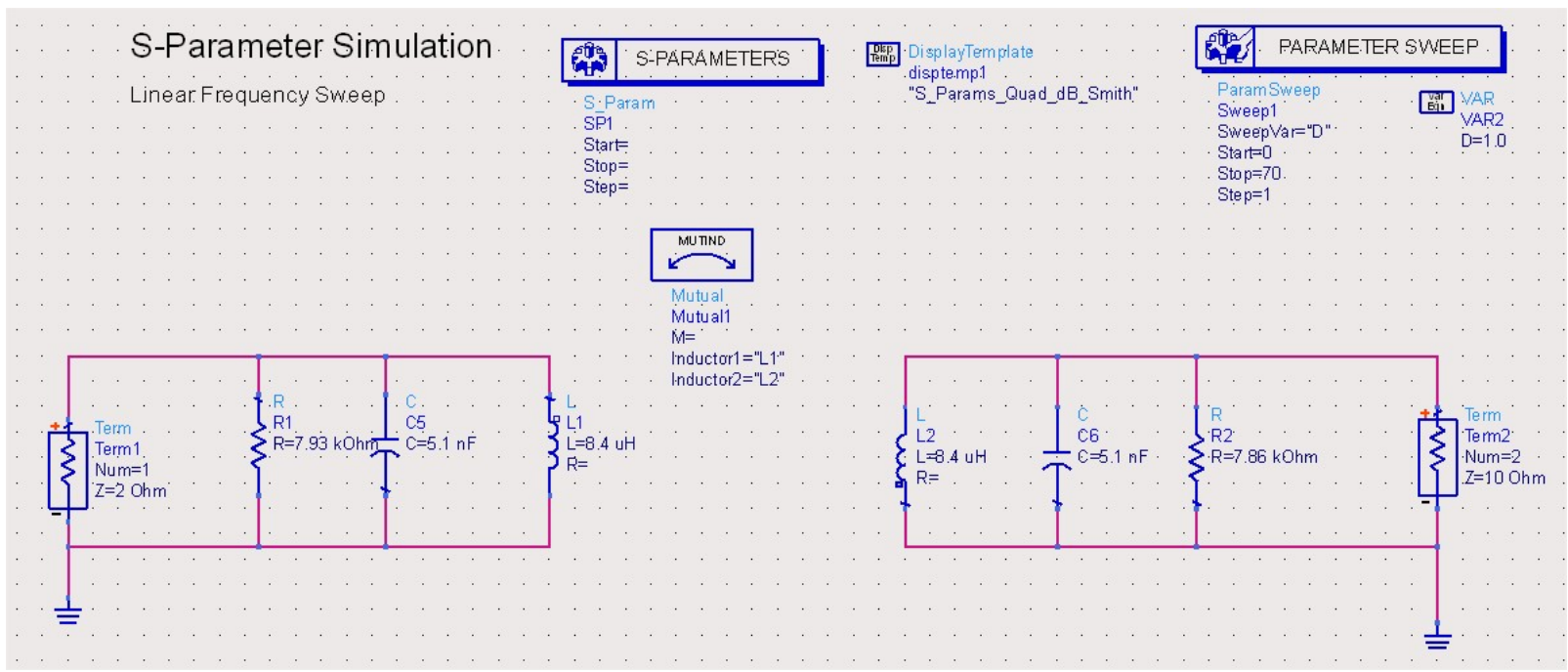

(a) Two coil unmatch system

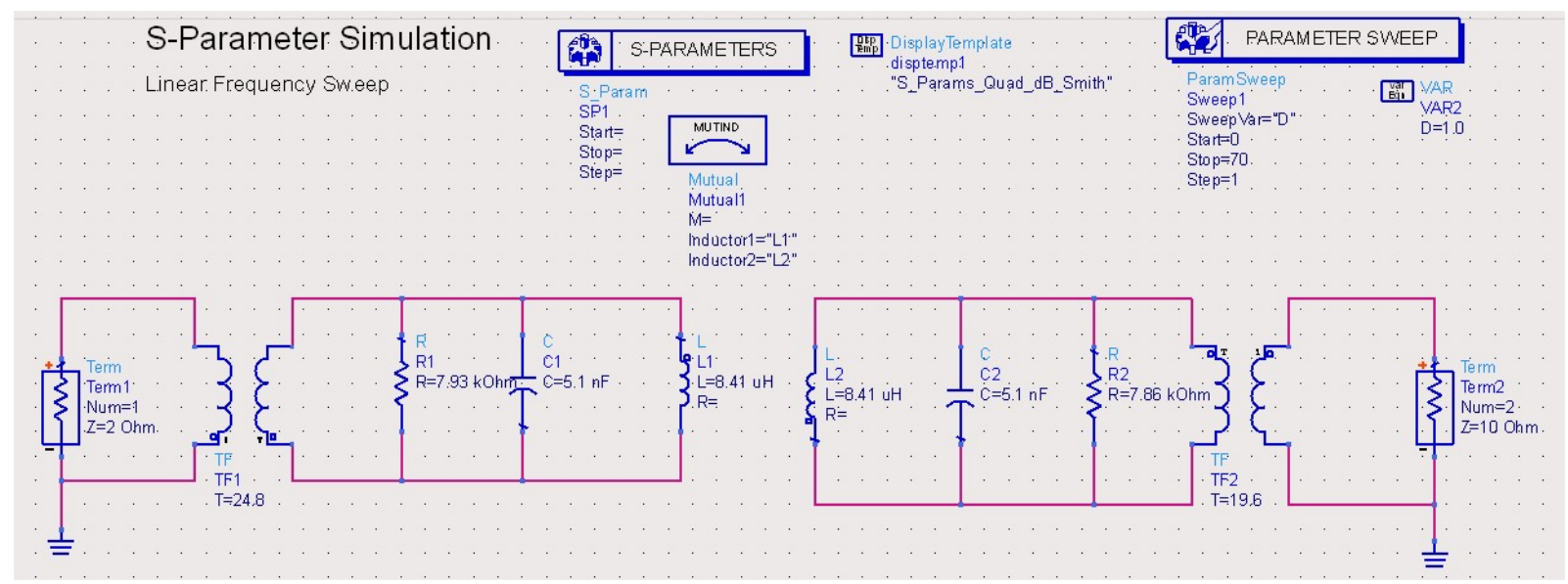

(b) Impedance matching two coil system using transformer

Fig. 2 Agilent ADS simulation schematics for $30 \mathrm{~cm}$ diameter coil.

\section{Experimental Setup}

Fig. 3 illustrated the connection between the power source, function generator and transmitter circuit. The transmitter circuit was then connected to a step-up transformer and to the transmitter. Another side of the receiver circuit consist of the receiver coil, capacitor, step-down transformer and load. The load was connected to the LCR meter to undergo result measurement.

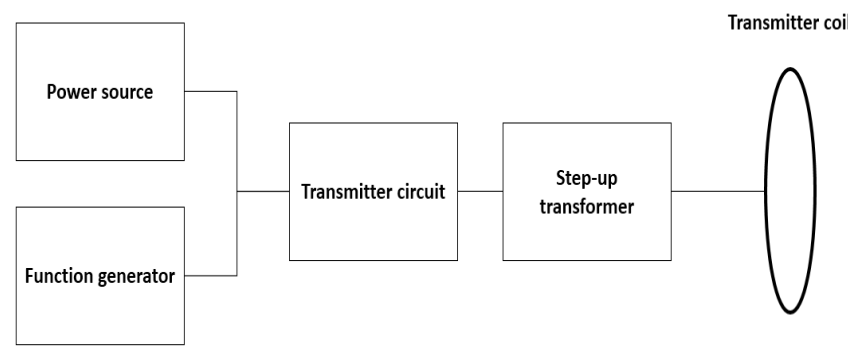

Fig. 3 Connection for power source and transmitter circuit.

Fig. 4 illustrated the connection between receiver circuit, load and oscilloscope.

\section{Receiver coil}

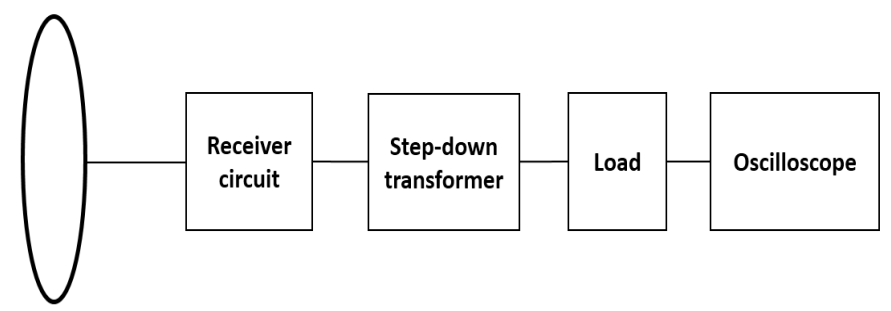

Fig. 4 Schematic diagram for preparation of $\mathrm{TiO}_{2} @ \mathrm{Ag}$ and $\mathrm{Ag} / \mathrm{TiO}_{2}$ microspheres.

The transmitter circuit was connected to the power source and function generator as shown in figure 5(a). The transmitter circuit was then connected to a step-up transformer and to the transmitter. Another side of the receiver circuit consist of the receiver coil, capacitor, step-down transformer and load. The load was connected to the LCR meter to undergo result measurement. Figure 5(b) shows the setup for receiver circuit with receiver coil and connected load with oscilloscope. The result was recorded every centimeter starting from $5 \mathrm{~cm}$ up to $70 \mathrm{~cm}$. After the result have been measured and recorded, it is compared with the simulated result using Agilent ADS to verify the efficiency. 


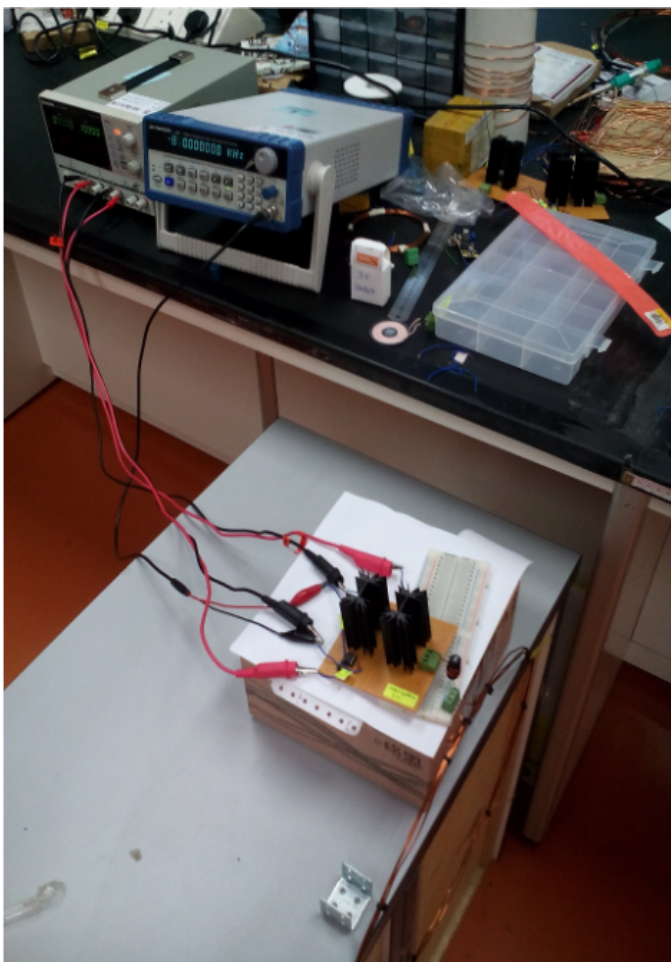

(a)

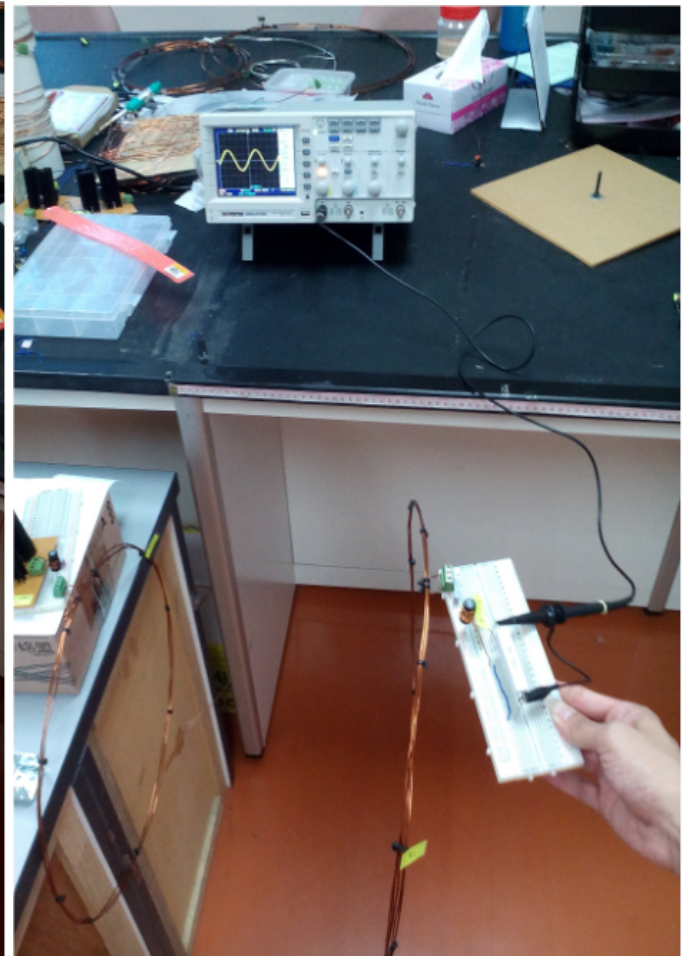

(b)

Fig. 5 (a):Transmitter circuit connect to function generator and power source, (b): Receiver circuit connect to oscilloscope.

\section{RESULTS AND DISCUSSION}

\section{Qualify Factor of The Transmitter and Receiver Coils}

This section decribes the design of the transmitter and receiver coils pair been designed. Several number of turns of the coils had been fabricated and the quality factor had been measured to obtain the number of turns with the best quality factor of the coils. Figure 6 shows the quality factor for the different number of turns of the coils during different resonant frequencies. The thickness of the coil is approximately $1.5 \mathrm{~mm}$. The results are measured and recorded using LCR meter. These results are serve as references during the fabrication process of transmitter and receiver coils pair. The quality factor is divided into four different category which is $250 \mathrm{kHz}$, $500 \mathrm{kHz}, 750 \mathrm{kHz}$ and $1 \mathrm{MkHz}$ to be measured.

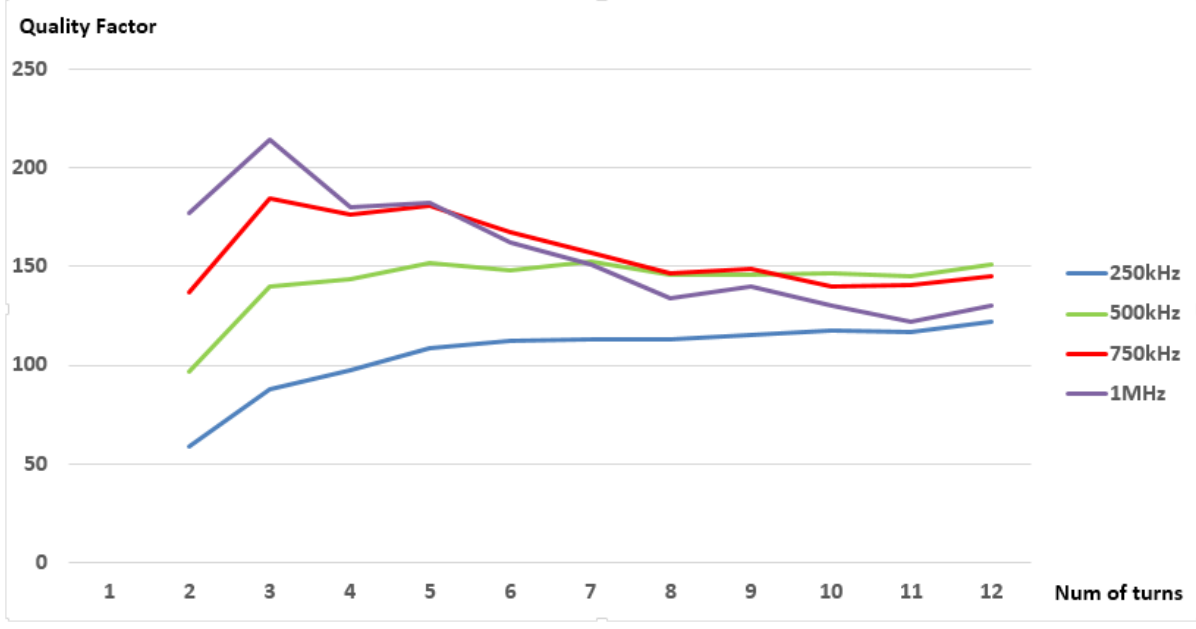

Fig. 6 Quality factor versus number of turns of $30 \mathrm{~cm}$ coils.

For different categories of frequency resonance, the turns of coils will be defferent. For resonant frequency at $250 \mathrm{kHz}$ and $500 \mathrm{kHz}$, the twelve turns of coil show the highest quality factor. For resonant frequency at $750 \mathrm{kHz}$ and $1 \mathrm{MHz}$, three turns of coil show the highest quality factor. During the experiment, the resonant frequency used for the $30 \mathrm{~cm}$ diameter coils pair was $784 \mathrm{kHz}$. The graph show the highest quality factor was 184.5 when the number of turns of coil equal to three. The graph also shows the quality factor of the coil decreased when the number of turns of the coil increased. So, three turns of coils is chosen to be implemented as transmitter and receiver coils to the system. It showes better performance compared to other number of turns coils.

\section{Simulation Results Comparison Before and After Impedance matching}

Agilent Advance Design System was used to perform simulation. The matched two coil WPT system and unmatched two coil WPT system were simulated for comparison. The parameter $S_{21}$ 
was measured to serve as the indicator for the system performance. The gain for both transformers, $n_{1}$ and $n_{2}$ were calculated. The purpose of doing the simulation is to ensure that the impedance matching method can help to improve the performance of the WPT system. Figure 7 shows the result comparison for before and after implementing impedance matching method for WPT system for $30 \mathrm{~cm}$ diameter coil transmitter and receiver pair.

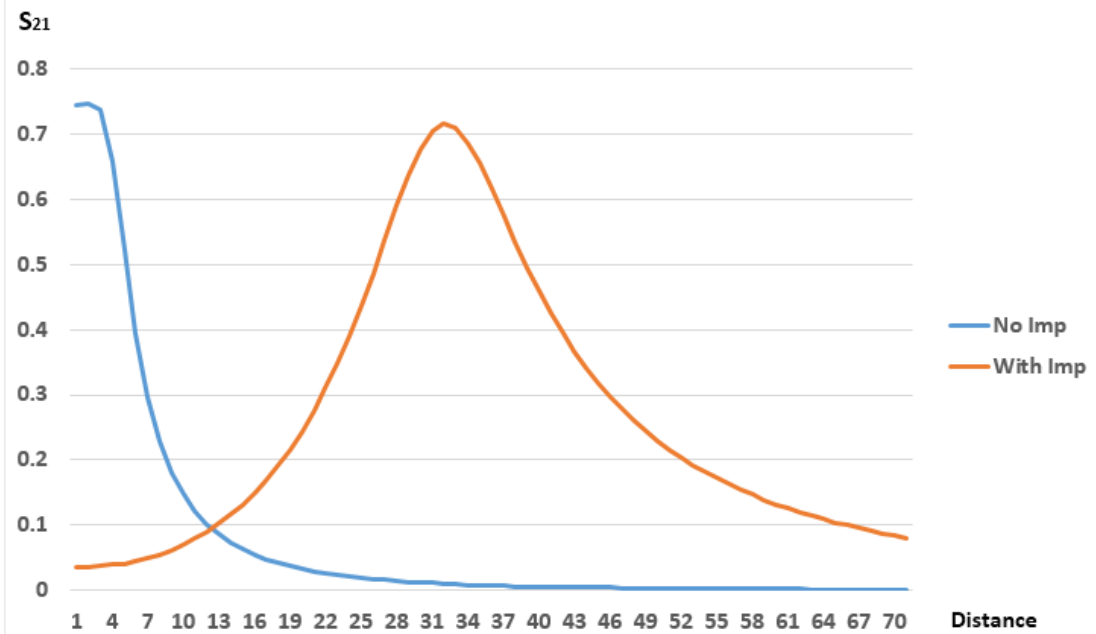

Fig. 7 Simulation results for match and unmatch impedance for $30 \mathrm{~cm}$ diameter coil

The blue colour line is the simulation result without any impedance matching method and the red colour line is the simulation result after implement the impedance matching method (with transformers). The unmatched two coil contactless power transfer system shows a very high efficency over a very short transmission distance. The highest efficiency recorded is 75 percent with transmission distance equal to $3 \mathrm{~cm}$. However the highest efficiency for the matched two coil contactless power transfer system is 71.6 percent with transmission distance equal to $31 \mathrm{~cm}$. The highest efficiency performed by both unmatched and matched system are almost similar.
However, it shows that the matched two coil contactless power transfer system for $30 \mathrm{~cm}$ diameter coil can increase approximately 10 times the transmission distance than the unmatched two coil contactless power transfer system. Moreover, the matched two coil contactless power transfer system can transmit up to $40 \mathrm{~cm}$ distance with 50 percent efficiency. The result shows that matched two coil contactless power transfer system for $30 \mathrm{~cm}$ transmitter and receiver pair can provide better performance.

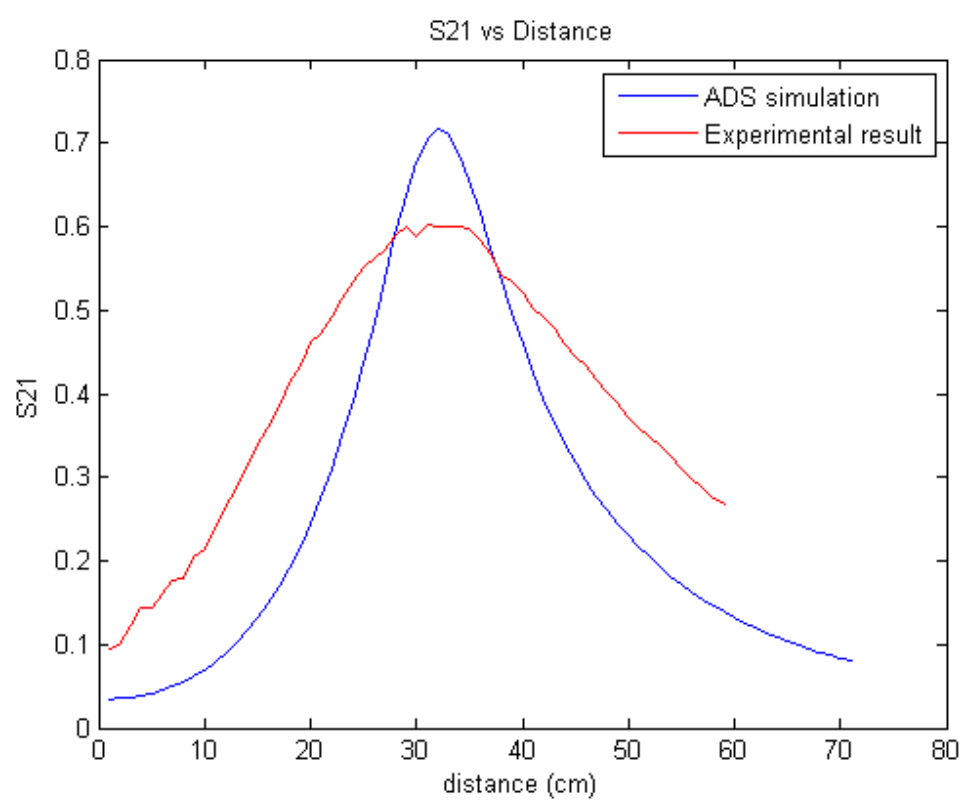

Fig. 8 Comparison for the experimental and simulation results for $30 \mathrm{~cm}$ diameter coil

\section{Comparison of Experimental Result and Simulation Result for $30 \mathrm{~cm}$ Coil Diameter}

Fig. 8 shows the comparison of the experimental result and the simulation result using Agilent Advance Design System. Based on Fig. 4, a transfer distance of $46 \mathrm{~cm}$ is achieved using magnetic coupling with two resonance coils of $30 \mathrm{~cm}$ diameter and able to yeild an energy transfer up 50 percent (experimental result) which is approximately 1.5 time of the coil diameter. However, it means that the contactless power transfer system can only maintain its high efficiency at a limited distances. The simulation result shows the highest efficiency of 70 percent at a distance of $32 \mathrm{~cm}$ and the

experimental result shows the highest efficiency of 60 percent at a distance of $35 \mathrm{~cm}$. The performance of this system decreases when the 
distance of transmitter and receiver coils increases. At a distance of $60 \mathrm{~cm}$, the system still manage to achieve 33 percent of transfer efficiency. The experimental result shows lower performance as compared to the simulated result. There are few reasons contribuding to this difference which are impedance mismatch and power losses in transformer.

The step-up and step-down transformers with gain, $n_{1}=24.8$ and $n_{2}=19.6$ respectively are designed manually. Therefore its gain will be slightly different from the simulation value. The difference in the transformers gain caused the impedance of the system to be slightly unmatched and the manually designed transformers cannot achieve the simulation ideal state. The second reason is the power loss from the transformer during the experiment. The temperature of the step up transformer in the transmitter circuit increased and changed the transformer resistance. The connection of the wires and connectors used in the transmitter and receiver circuit also caused the loss of power during experiment which lead to the difference between the experimental result and simulation result.

However, this contactless power transfer system demostrates the approach for matching the system impedance correctly without any aid of the multiple resonators to optimize the performance of the system. In addition, the temperature of transmitter and receiver remain unchange throughout the experiment. Therefore this two coil wireless power transfer system can be implemented to other practical medical charging devices.

\section{CONCLUSION}

A study of contactless power transfer system for low power medical devices with magnetic coupling technique has been demonstrated. The contactless power transfer system adopted the two coil wireless power transfer system to operate. It successfully transmitted the power wirelessly through the transmitter circuit to the receiver circuit. The transfer distance achieved by the coils with diameter with $30 \mathrm{~cm}$ with highest transfer efficiency recorded approximately 1.1 time its coil diameters $(32 \mathrm{~cm})$. An innovative impedance matching for both transmitter and receiver circuit by adding a step-up and step-down transformers has been proposed. From the simulation, it shows that the impedance matching method improved the transfer efficiency and distance of the contactless power transfer system for low power medical devices. This impedance matching method is universal and can be applied to all the two coil contactless power transfer system. This impedance matching method also help to reduce the size of the contactless power transfer system because it can work without any help of the resonators such as the four coils wireless power transfer system. The high quality factor of the transmitter and receiver coils has been chosen to optimize the transfer efficiency of the system. Three number of turns are the ideal number of turns for the transmitter and receiver coils according to the simulation.

\section{ACKNOWLEDGEMENT}

This research is a collaboration between Universiti Teknologi Malaysia (UTM) and Institute Jantung Negara (IJN). The authors gratefully acknowledge the research grant provided to this study by Research Management Centre sponsored by Ministry of Education, Malaysia, Vot: 16h19 (Research University Grant), Universiti Teknologi Malaysia, Johor Bahru, Malaysia.

\section{REFERENCES}

Badr, B. M., Somogyi-Csizmazia, R., Leslie, P., Delaney, K. R., and Dechev, N., 2017. Design of a wireless measurement system for use in wireless power transfer applications for implants. Wireless Power Transfer, 4(1) : 21-32.

Bawa, G., Ghovanloo, M. 2008. Active high power conversion efficiency rectifier with built-in dual-mode back telemetry in standard CMOS technology. IEEE Transactions on Biomedical Circuits and Systems, 2(3): 184-192.
Chandrakasan, Anantha P., Naveen Verma, Denis C. Daly. 2008. Ultralowpower electronics for biomedical applications. Annual Review of Biomedical Engineering, 10: 247-274.

Hui S. Y., Zhong W., Lee C. K. 2014. A critical review of recent progress in mid-range wireless power transfer. IEEE Transactions on Power Electronics, 29(9): 4500-4511.

Imura, T. and Hori, Y 2011. Maximizing air gap and efficiency of magnetic resonant coupling for wireless power transfer using equivalent circuit and Neumann formula. IEEE Transactions on Industrial Electronics, 58(10): 4746-4752.

Leong K. M., Lum K. Y., Tan T. S., Md Khudzari, A. Z., Suhaini, K. 2016. Wireless Power Transfer System for Biomedical Devices by using Magnetic Resonance Coupling Technique. Research Journal of Applied Sciences, Engineering and Technology, 12(8): 823-827.

Lee, C. K., Zhong, W. X., Hui, S. Y. R. 2012. Effects of magnetic coupling of nonadjacent resonators on wireless power domino-resonator systems. IEEE Transactions on Power Electronics, 27(4): 1905-1916.

Liu, F., Yang, Y., Jiang, D., Ruan, X., Chen, X. 2017. Modeling and optimization of magnetically coupled resonant wireless power transfer system with varying spatial scales. IEEE Transactions on Power Electronics, 32(4): 3240-3250.

Mur-Miranda, J. O., Fanti, G., Feng, Y., Omanakuttan, K., Ongie, R., Setjoadi, A., Sharpe, N. 2010. Wireless power transfer using weakly coupled magnetostatic resonators. Energy Conversion Congress and Exposition (ECCE) 2010 IEEE. 12-16 September 2010. Hilton Atlanta Atlanta, USA: IEEE, 4179-4186.

RamRakhyani, A. K., Mirabbasi, S. and Chiao, M., 2011. Design and optimization of resonance-based efficient wireless power delivery systems for biomedical implants. IEEE Transactions on Biomedical Circuits and Systems, 5(1): 48-63.

Samad, N. A., Vaithianathan, T., Aziz, S. M., Brander, C. E., 2006. Design of a wireless power supply receiver for biomedical applications. Asia Pacific Conference on Circuits and Systems (APCCAS) 2006. 4 -7 December 2006. Grand Copthorne Waterfront Hotel, Singapore, 674-677.

Si, P., Hu, A. P., Hsu, J. W., Chiang, M., Wang, Y., Malpas, S., Budgett, D., 2007. Wireless power supply for implantable biomedical device based on primary input voltage regulation. 2nd IEEE Conference on Industrial Electronics and Applications (ICIEA), 23-25 May 2007. Harbin, China: IEEE, 235-239.

Slaughter, Mark S., and Timothy J. Myers. 2010. Transcutaneous energy transmission for mechanical circulatory support systems: history, current status, and future prospects. Journal of Cardiac Surgery, 25(4): 484-489.

Syms, R. R. A., Solymar, L., Young, I. R., Floume, T. 2010. Thin-film magneto-inductive cables. Journal of Physics D: Applied Physics, 43(5), 055102 .

Wang, B., Hu, A. P., Budgett, D., 2013. Autonomous synchronous rectifier for heart pump applications. In 2013 IEEE International Conference on Industrial Technology (ICIT 2013). 25-28 February 2013. Western Cape, South Africa: IEEE, 481-486.

Waters, B. H., Sample, A. P., Bonde, P., Smith, J., 2012. Powering a ventricular assist device (VAD) with the free-range resonant electrical energy delivery (FREE-D) system. Proceedings of the IEEE, January 2012, 100(1): 138-149.

Waters, Benjamin H., Joshua R. Smith, and Pramod Bonde. 2014. Innovative free-range resonant electrical energy delivery system (FREE-D system) for a ventricular assist device using wireless power. ASAIO Journal, 60(1) :31-37.

Zhang, F., Hackworth, S. A., Fu, W., Sun, M. 2010,. The relay effect on wireless power transfer using witricity. In 14th Biennial IEEE Conference on Electromagnetic Field Computation (CEFC) 2010. 09-12 May 2010. Chicago, USA: IEEE, 1-1.

Zhang, F., Hackworth, S. A., Fu, W., Li, C., Mao, Z., Sun, M., 2011. Relay effect of wireless power transfer using strongly coupled magnetic resonances. IEEE Transactions on Magnetics, 47(5): 1478-1481.

Zhong, W. X., Lee, C. K., Hui, S. Y., 2012. Wireless power domino-resonator systems with noncoaxial axes and circular structures. IEEE Transactions on Power Electronics, 27(11): 4750-4762.

Zhong, W., Lee, C. K., Hui, S. R., 2013. General analysis on the use of Tesla's resonators in domino forms for wireless power transfer. IEEE Transactions on Industrial Electronics, 60(1): 261-270. 